\title{
Nucleation and crystallization in aqueous systems during drying: Theory and practice
}

\author{
Felix Franks and Norio Murase ${ }^{+}$ \\ Biopreservation Division, Pafra Ltd., 150 Cambridge Science Park, \\ Cambridge CB4 4GG, U.K. \\ +permanent address: Laboratory of Chemistry, Tokyo Denki University, \\ Saitama 350 03, Japan
}

\begin{abstract}
The actual phase behaviour of alkali metal phosphates during the freeze and air drying of aqueous solutions has been studied and compared to the predictions based on the equilibrium phase diagrams. For the ternary sodium phosphate + water system, the ternary eutectic concentration ratio is far removed from the concentration ratio corresponding to $\mathrm{pH} 7$. It has been found that under conditions of freezing, two of the four phosphate salts studied, precipitate rapidly, but not completely, at the eutectic temperature, one sait is subject to a low rate of nucleation and $\mathrm{NaH}_{2} \mathrm{PO}_{4}$ does not precipitate at all. In every case the degree of precipitation increases with the initial sait concentration. In ternary mixtures, containing sucrose, salt and a low residual moisture content, the salts form a solid solution with sucrose and can be made to crystallise slowly from the amorphous phase as the temperature is raised slightly above the glass transition temperature of the mixture.
\end{abstract}

\section{INTRODUCTION}

During the past few years a general realisation has emerged of the importance of the properties of water soluble or water sensitive amorphous solids in many branches of technology (1). For instance, demands for improved stabilisation techniques have followed the isolation of labile bioproducts, many of them highly purified proteins. Most proteins in dilute aqueous solution are readily inactivated; only few methods are available for improving the long-term stability of such products (2); they all rely on the removal of upwards of $90 \%$ of the water. Despite its high cost in capital and energy and the necessity for long processing times, freeze-drying (lyophilisation) has established itself as the preferred method for protein stabilisation, especially in the pharmaceutical and biotechnology industries (3). However, the freeze-drying process is rarely performed to a sufficiently high standard for its inherent advantages to be fully exploited (4). Protein activity losses during freeze-drying tend to be substantial, and further losses can occur during subsequent storage, even if the oried product is kept at $-20^{\circ} \mathrm{C}$. Recent fundamental investigations into the processes that accompany the removal of water from an aqueous solution have revealed the importance of eutectic phenomena, supersaturation, the properties of water as a plasticiser for natural materials and slow crystallisation processes within amorphous substrates to an achievement of stability in labile products.

\section{CRYSTALLINE AND AMORPHOUS STATES}

A crucial factor which determines the shelf-life of a dried biological product is its final physical state, regardless of the processing method by which water was removed. solids can exist in crystalline and amorphous forms. Crystalline materials have defined stoichiometric compositions and structures and are considered to be thermodynamically stable within wel1-defined ranges of temperature, pressure and composition. An amorphous material, by contrast, has no clearly defined molecular structure. It is 
obtained from the liquid by rapid cooling. In principle, a liquid should freeze (crystallise) on cooling, but this does not always happen in practice. Under conditions of "rapid" cooling (the term "rapid" is here used only to describe the rate of cooling relative to the rate of crystallisation) 7 iquid states can often persist well belon' their normal freezing points. As cooling continues, so the viscosity of the supercooled liquid rises increasingly steeply with decreasing temperature. The initially mobile fluid turns first into a syrup, then into a viscoelastic "rubber", a deformable "leather" and finally into a brittle "glass", without ever crystailising. A glass is therefore a supercooled liquid with an extremely high viscosity. As such, it is thermodynamically unstable but derives kinetic stability from its high viscosity. Viscous flow in a glass is of the order of $\mathrm{m} /$ century. Mechanically, if not structurally, glasses are therefore solids.

\section{THE GLASS TRANSITION}

The transition from a deformabie viscoelastic "rubber" to a glass takes place over a relatively narrow temperature interval where the viscosity rises by several orders of magnitude over a temperature interval of only a few degrees. The physical properties of inorganic glasses (metal alloys, silicates) and synthetic polymer/plasticiser systems have been studied in great detail (5), but this is hardly the case for water-sensitive products, especially those obtained from initially dilute solution by the freezing-out or evaporation of water. Essentially the problems center on the physical and chemical changes which can occur during and after the conversion of an aqueous solution of low solid content (say $5 \%$ by weight) into a solid of low water content.

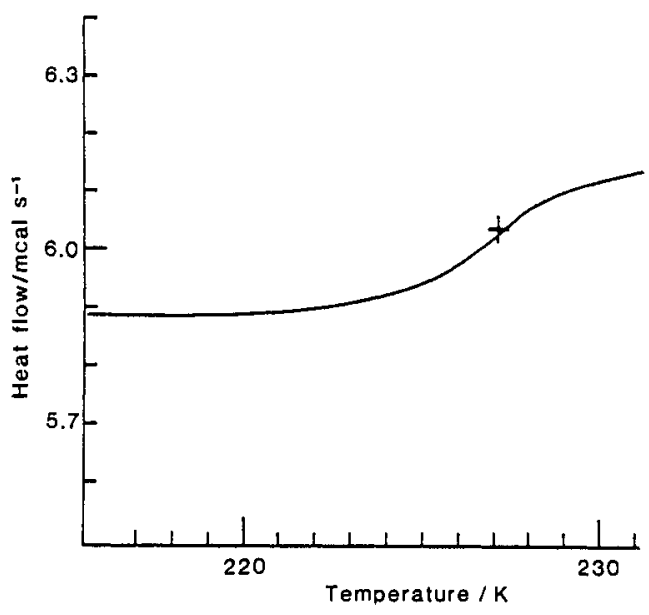

Figure 1. DSC heating trace for a frozen solution containing sucrose and $\mathrm{NaCl}$, showing the glass/rubber transition of the freeze concentrate (D in Fig. 2) at 227K.

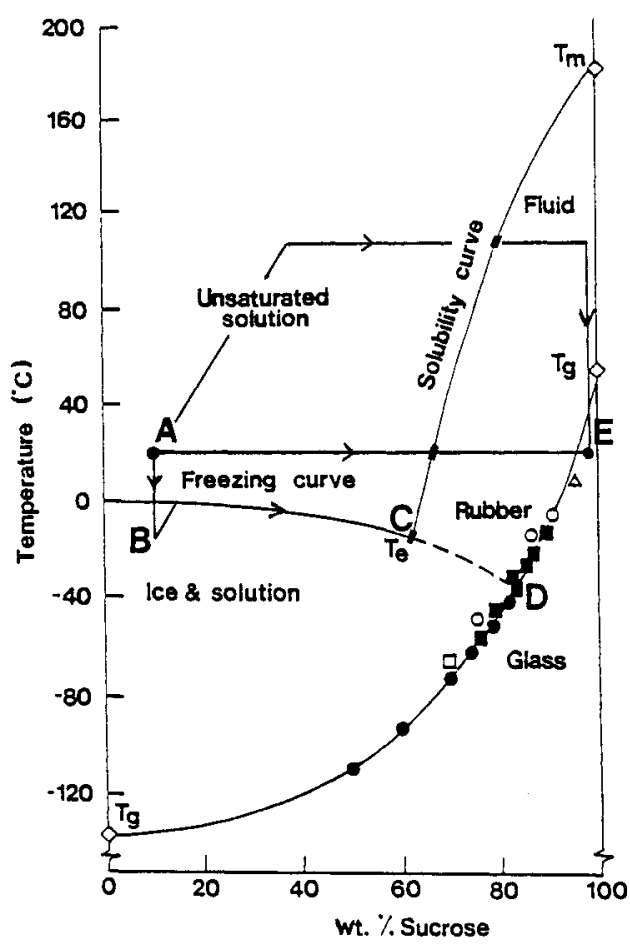

Figure 2. Solid-7iquid state diagram, showing different paths for the removal of water from a dilute solution (A), to a solid (E); for details see text.

The glass transition can be identified experimentally by various techniques, most popular among them differential scanning calorimetry, DSC (6). If a glassy material which has been obtained by quenching a liquid, is heated at a constant rate, then its specific heat, measured as a function of temperature, exhibits a discontinuity, as shown in Fig. 1 for a mixture containing sucrose and $\mathrm{NaCl}$. The centre of the transition is identified with $\mathrm{Tg}$. The glass transition temperature depends on the composition of the amorphous phase and is particuiarly sensitive to its moisture content $w$; this is 
shown for the sucrose-water system in Fig. 2. The $T_{g}(w)$ profile therefore defines the practical limit of the product stability region. It must be emphasised that the $\mathrm{Tg}(\mathrm{W})$ curve is not a phase boundary but merely an isoviscosity locus.

Figure 2 also shows the freezing point locus $A B C D$ which ends at $T g$ and the eutectic point $C$ at which, under equilibrium conditions, the solute crystallises. In a binary system the solidus curve joins the melting point of the solute and $T_{e}$. In a multicomponent system $T_{e}$ correponds to the first eutectic encountered along the liquidus curve. Finally, Fig. 2 also illustrates other possible paths which can be chosen for the removal of water, by evaporation. They all have to cross the solidus curve at some point, when precipitation can, in principle, take place.

\section{KINETICS IN AMORPHOUS SYSTEMS}

The flow rates of mobile fluids and the elastic moduli of solids exhibit temperature dependencies which are adequately described by the Arrhenius relationship. In the rubber region (near the glass transition), however, the dynamic properties of the material are better represented by various other semi-empirical relationships, one of which is the Williams-Lande1-Ferry (WLF) equation:

$$
\text { ln } k=-C_{1}\left(T-T_{g}\right) /\left[C_{2}+\left(T-T_{g}\right)\right]
$$

where $k$ is a a rate constant (e.g. for viscous flow), $C_{1}$ and $C_{2}$ are universal constants and $\mathrm{T}_{\mathrm{g}}$ is the glass temperature. It follows from eqn. (1), that in the proximity of the glass transition, $k$ is much more sensitive to the temperature than is predicted by the Arrhenius equation (1). Equation (1) also demonstrates that the rate of flow (or diffusion) is governed by the difference between the actual temperature and the glass transition temperature $\left(T-T_{g}\right)$. This is illustrated in Fig. 3 , where WLF kinetics are compared with conventional Arrhenius kinetics.

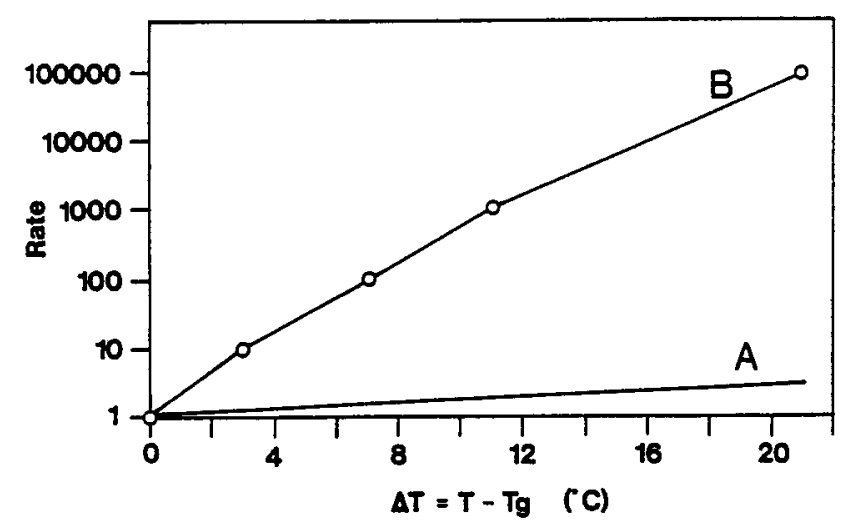

Figure 3. Relative dependence of kinetic rate processes on $\left(T-T_{g}\right.$ ) for A: typical Arrhenius fluids and solids (with an activation energy of $80 \mathrm{~kJ} / \mathrm{mol}$ ) and B: rubbery materials in which rates follow WLF kinetics.

Most chemical and biochemical reactions which might affect the shelf life of a labile product are also governed by WLF kinetics, in an environment of a rubbery nature, i.e. in a very concentrated mixture. Whether the crystallisation of salts or other components from "rubbers" is also governed by WLF kinetics remains to be established.

\section{SOLUTE CRYSTALLISATION DURING FREEZING}

Under equilibrium conditions the course of freeze concentration in a multicomponent mixture is determined by the colligative freezing point depression. In the 1 imit of $c 0$, the slope of the liquidus curve $T$ fc) is given by the cryoscopic constant (1.86 $\mathrm{deg} / \mathrm{mol}$ ). The curvature depends on the nonideality of the solution (solute-solute interactions). While the nucleation and crystal growth kinetics of ice have been described in considerable detail ( 7 ) and continue to be a subject of great interest (8), the phase separation of saits and other substances during (or after) freezing is still quite unexplored. 
The equilibrium freezing point curve terminates at the first binary eutectic point $T_{e}$, provided that the solute in question actually crystallises at this temperature (see below). This point is important when $\mathrm{pH}$ buffer mixtures are considered. The eutectic data for sodium and potassium phosphates are summarized in Table 1. For the sodium salts at the ternary eutectic temperature, the mol ratio $\mathrm{NaH}_{2} \mathrm{P} / \mathrm{Na}_{2} \mathrm{HP}=57$. Any mixture that contains the salts in another ratio should suffer pH shifts if one or the other of the salts crystallises as a binary eutectic mixture with ice during cooling. However, the mol ratio for a $\mathrm{pH} 7$ buffer is 0.72 . The eutectic concentration ratio is therefore not appropriate for $\mathrm{pH}$ buffering of proteins, because large $\mathrm{pH}$ changes would be expected to occur during freezing. The mol ratio for the potassium salt ternary eutectic is 0.48 , with $\mathrm{T}_{e}=-16.7^{\circ} \mathrm{C}$, $i . e$. much closer to the mol ratio corresponding to $\mathrm{pH} 7$ and of a better freeze stability than the $\mathrm{Na}$ salt. In the presence of other, neutral salts, quaternary eutectics (three salts + ice) can be formed with different composition ratios. Even if a protein was not damaged by excessive pH shifts during freezing, the increasing buffer salt concentration is likely to lead to concentration denaturation (4).

Table 1. Eutectic data of phosphate solutions: (1) monosodium (potassium) salt, (2) disodium (potassium) sait.

\begin{tabular}{lllll}
\hline & $T_{e}^{0}$ & $C_{e}(1)(M)$ & $C_{e}(2)(M)$ & $C_{e}(1) / C_{e}(2)$ \\
\hline $\mathrm{NaH}_{2} \mathrm{PO}_{4}$ & -9.7 & 3.42 & & \\
$\mathrm{Na}_{2} \mathrm{HPO}_{4}$ & -0.5 & 3.42 & 0.11 & $57(0.72)^{a}$ \\
$\mathrm{NaH}_{2} \mathrm{PO}_{4}-\mathrm{Na}_{2} \mathrm{HPO}_{4}$ & -9.9 & 0.92 & & \\
$\mathrm{KH}_{2} \mathrm{PO}_{4}$ & -2.7 & -13.7 & & \\
$\mathrm{~K}_{2} \mathrm{HPO}_{4}$ & -16.7 & 1.30 & 2.85 & $0.48(0.72)^{a}$ \\
$\mathrm{KH}_{2} \mathrm{PO}_{4}-\mathrm{K}_{2} \mathrm{HPO}_{4}$ & & & & \\
\hline
\end{tabular}

a corresponds to the $\mathrm{pH} 7$ buffer ratio

\section{CRYSTALLISATION OR SUPERSATURATION}

As distinct from predictions based on the equilibrium phase behaviour, the actual crystallisation of buffers and other solution components during freezing depends on the sample size, the cooling rate and the chemical nature of the solutes, their initial concentrations and nucleation rates in the mixture. Under freezing conditions, salt crystal nucleation and growth are very slow compared to ice crystal growth. Freeze concentration therefore proceeds rapidly and the residual unfrozen solution phase tends to be compartmentalized in the form of microdomains between the advancing ice crystals. The processes accompanying freeze concentration have been quantitatively analysed by Körber (9). The probability of salt nucleation within the supersaturated microdomains depends in a complex manner on several factors, including their volumes, the initial and eutectic concentrations and the cooling rate. In binary phosphate solutions subjected to slow freezing ( $5 \mathrm{deg} / \mathrm{min}$ ), $\mathrm{KH}_{2} \mathrm{PO}_{4}$ and $\mathrm{NaH}_{2} \mathrm{PO}_{4}$ have been found to crystallise rapidly, $\mathrm{K}_{2} \mathrm{HPO}_{4}$ crystallises slowly and $\mathrm{NaH}_{2} \mathrm{PO}_{4}$ does not crystallise at all (10). Using as criterion the concentration dependence of the eutectic melting enthalpy, it has been found, as shown in Fig. 4, that comolete crystallisation of salts from binary freezing solutions does not occur in solutions of $c<500 \mathrm{mM}$, such as are commonly used in biochemical preparations. This is the case even for $\mathrm{NaCl} .2 \mathrm{H}_{2} \mathrm{O}$. Furthermore, no ternary phosphate eutectic phase separation is observed in mixed phosphate solutions at any initial concentration. Instead, the addition of $\mathrm{K}_{2} \mathrm{HPO}_{4}$ inhibits the crystallisation of $\mathrm{KH}_{2} \mathrm{PO}_{4}$, and vice versa, as shown in Fig. 5 . The results shown in Figs. 4 and 5 have been confirmed by scanning electron microscopy of the freeze-dried solution (11).

It is therefore common for frozen salt solutions to become supersaturated either completely or partiy. Supersaturation can sometimes be prevented by annealing the frozen mixture, i.e. by temperature cycling below the freezing point. Salt crystallisation which is inhibited wholly or partially during freezing will frequently take place when the frozen mixture is heated, even after the ice has been sublimed. It 


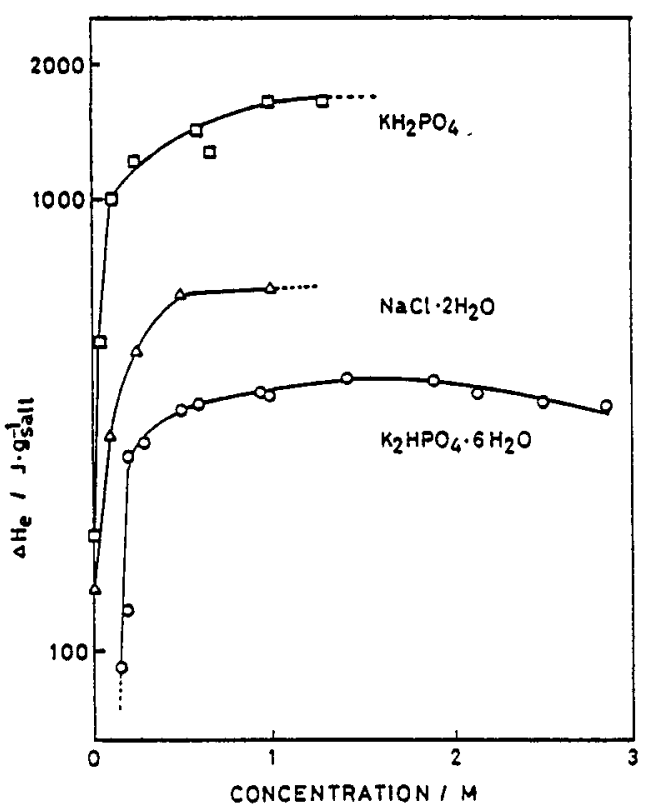

Figure 4. Dependence of the heat of eutectic melting on the salt concentration.

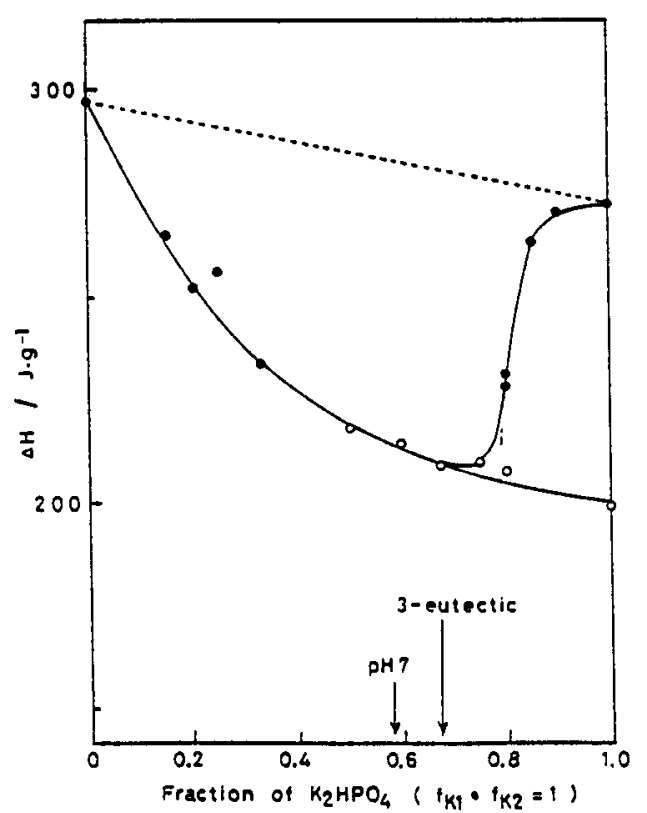

Figure 5. Heat of melting of $1 \mathrm{M}$ potassium phosphate buffers. Broken line: additive contributions to $\triangle H$. Arrows indicate the the compositions of the ternary eutectic and the $\mathrm{pH} 7$ buffer, repectively.

should, however, be noted that the aim in freezing processes is frequentiy to achieve supersaturation of some solutes. This is in fact the very basis of action of additives as cryoprotectants and stabilisers (excipients) during freeze drying (3).

\section{SALT CRYSTALLISATION FROM AMORPHOUS SOLIDS}

During the drying of labile biochemical preparation, the active component seldom crystaliises. At the end of the process, shown by $E$ in Fig. 2, it forms part of the amorphous phase, and in order to achieve a maximum shelf 1 ife and insensitivity to residual moisture, the aim should be to formulate the product in such a way as to maximise the amorphous phase mass fraction which should also have a high $T_{g}$.

Because of the thermodynamic instability of amorphous mixtures, they are vulinerable to phase separation, if the temperature is allowed to exceed $\mathrm{Tg}$ for a length of time or if the $\mathrm{T}_{\mathrm{g}}(w)$ curve is crossed under isothermal conditions by the admission of moisture. Mode 1 mixtures of dry sugars (12) and of water, sucrose and $\mathrm{NaCl}$ (13) have been used to investigate the phase separation from dried preparations of zero or low residual

moisture content. In the binary anhydrous sugar blends, the sugar with the lower $\mathrm{T}_{\mathrm{g}}$ is found to act as diluent, depressing the $\mathrm{T}_{\mathrm{g}}$ of the other sugar, but no phase separation has been observed. In the ternary water/sucrose/NaCl mixture, the resulting end product is a solution of $\mathrm{NaCl}$ in sucrose, i.e. sucrose has taken the place of water as solvent. If the temperature is allowed to rise above the $T_{g}$ of the sucrose/salt solid solution, $\mathrm{NaCl}$ is found to crystallise slowly, with a surprisingly homodisperse crystal size distribution. In principle, $\mathrm{NaCl}$ should have a finite equilibrium solubility in sucrose and exhibit eutectic behaviour, although, in practice, it may not be possible to achieve the crystallisation of sucrose under these conditions. We do suggest, however, that solutions of salts in amorphous carbohydrate solvents, the viscosity of which can be sensitively adjusted, should form useful systems for studies of the controlled nucleation and crystal growth behaviour of electrolytes.

\section{Acknowledgements}

The authors wish to express their gratitude to Dr. P. Echlin of the Department of Botany, University of Cambridge for his active interest in this work and N.M. also wishes to thank the Fellows of Clare Hall for a Visiting Associateship during his Sabbatical in Cambridge. 


\section{REFERENCES}

1. H. Levine and L. Slade, Water Sci. Rev. 3, 79-185(1988).

2. F. Franks, in Characterization of Proteins (F. Franks, ed.), Humana Press, Clifton N.J., 1988, p. 95. Second edition in preparation.

3. M.J. Pika1, Biopharm 3, 18-27(1990).

4. F. Franks, Cryo-Letters 11, 93-110(1990).

5. S.R. Elliott, Physics of Amorphous Materials, Longman Scientific \& Technical, Harlow, 2nd ed., 1990.

6. J.L. Ford and P. Timmins, Pharmaceutica7 Therma 7 Analysis, E11 is Horwood Ltd., Chichester, 1989.

7. P. Hobbs, Ice Physics, Clarendon Press, Oxford, 1974,

8. D. Clausse, in Encyclopaedia of Emulsion Technology, Vol. 2, (P. Becher, ed.), Marcel Dekker, New York, 1989, p. 77.

9. Ch. Körber, Quat. Rev. Biophys. 21, 229-298(1988).

10. N. Murase and F. Franks, Biophys. Chem. 34, 293-300(1989).

11. N. Murase, P. Echl in and F. Franks, Cryobiology 28, 364-375(1991).

12. L. Finegold, F. Franks and R.H.M. Hatley, J. Chem.Soc. Faraday Trans. I 8, 29452951(1989).

13. C. Van den Berg, F. Franks and P. Echlin (in preparation). 